\title{
INTERVISTA AD ANDREA ZANZOTTO
}

\author{
Vera Lúcia de OLIVEIRA*
}

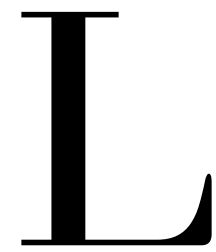

'intervista che segue è stata rilasciata a me a Perugia, nel 1993, e pubblicata per la prima volta nella rivista brasiliana Insieme, dell'Associazione di Professori di Italiano di San Paolo, e poi anche nel sito http://www. veraluciadeoliveira.it, curato da Claudio Maccherani. Quasi due decenni sono passati ed essa, grazie alla grande forza di vita e all'intensità di uno dei più grandi poeti italiani viventi, mantiene tutta la sua pregnanza e attualità. Recentemente questa intervista è stata "riscoperta" dalla critica italiana, perché praticamente non era conosciuta in questo paese, ed è stata inserita nel volume Con dolce curiosità: tributo ad Andrea Zanzotto, a cura di Matteo Chiavarone, libro che è un omaggio al poeta di Pieve di Soligo.

\footnotetext{
* Università degli Studi di Perugia
} 
Spesso i poeti hanno rapporti difficili con la propria città, la propria terra, un rapporto di amore-odio intenso e sofferto. Lei, che legami ha con Pieve di Soligo?

È un rapporto molto ambiguo, perché io sono nato in questo paesello prealpino veneto, sono sempre vissuto qui, mi sono spostato poco e sempre meno volentieri, con il passare degli anni. Questo fondo di angolo nascosto mi dava la possibilità di restare in una specie di rifugio "dalla storia". Era un rifugio non certo del tutto sotterraneo, ma formato di cunicoli che potevano condurre all'aperto o al chiuso a seconda delle esigenze di riparo, entro la mobilità onirica di quel caos che tutti abbiamo in noi.

Lo stare, il dimorare, l'immorare in un luogo unico, lo vedo d'altronde come semplice residuo di quella realtà contadina nella quale pochissimi, a meno che non fossero costretti da necessità di migrazione o fame o guerra, si muovevano. Si andava a fare il viaggio di nozze (qui da noi) magari fino a Treviso o a Venezia, e ciò quando già si era abbastanza in su nella gerarchia sociale.

Credo di aver ereditato certi pattern comportamentali da questo mondo contadino perché non sento un gran desiderio di muovermi, fermo restando che potrei amare svisceratamente qualunque luogo del mondo, perché la natura è sempre ricca di meraviglie, di paesi e di paesaggi capaci di ogni diversa seduzione, e di luoghi degni di viverci e di "essere sognati".

$\mathrm{Ma}$, penso, il rapporto con il luogo e con la terra deve essere feroce ed esclusivo, come un innamoramento, altrimenti non possiamo capire niente né di noi, né della terra, né dell'ambiente, né dell'universo. Ho già avuto modo di parlare di alienazione turistica. C'è gente che viene portata di qua e di là come sacchi di patate, mentre occorrerebbe quel voyage o grand tour che era possibile fino a tempi abbastanza recenti, una grande avventura di formazione spirituale che durava mesi o anni (però soltanto per pochi privilegiati).

Un'altra possibilità è il piccolo voyage, o meglio trip (e talvolta grande trip) per il quale non ho bisogno d'incentivazioni, perché posso compierlo in continuazione. Posso ripercorrere all'infinito le stesse strade, gli stessi minimi itinerari che, pur in perenne mutazione, nella loro apparente stabilità testimoniano i misteri della continua metamorfosi della natura. Devo aggiungere che per me contò sempre moltissimo la vicinanza di Venezia, dove "tutti" finiscono per arrivare, rendendomi possibile i più straordinari incontri di letterati e artisti di tutto il mondo.

Ma poi, anche questo rapporto con la propria terra, come tutti gli amori, è destinato a finire: ad un certo momento ci si sente lontani da tutto. Alle volte penso che abbandonerei volentieri il mio paese, anche perché più si diventa vecchi più si vedono scomparire attorno a noi i compagni di vita. Si diventa un po' sconosciuti, e io per parte mia comincio già a conoscere poco i giovani 
del paese. Continuo ad andare di tanto in tanto nelle scuole, però ho in prevalenza rapporti con quei giovani e con quegli insegnanti che vengono a cercarmi perché hanno vivi interessi per la letteratura e la scuola.

\section{Com'è stata la sua infanzia? È stata felice? E quanto questo suo attaccamento e amore per la natura hanno inciso nel suo modo di essere e nel suo fare poesia?}

Già nella lontana infanzia, mi fu duro avvertire la situazione anomala della mia famiglia, in lotta con la precarietà. Si era reso difficilissimo il lavoro a mio padre per la sua opposizione al regime. Poteva mancare da un giorno all'altro il sostentamento. Si apparteneva a una fascia piccolo-borghese ma quasi di miseria. Mio padre ha spesso dovuto cercare lavoro all'estero o in zone difficili dell'Italia, come l'alto Cadore. Nel nostro paese pochi avevano votato contro il fascio nel plebiscito del 1929 e, fra questi, c'era mio padre, cosa che tutti sapevano. Ricordo che la maestra a scuola ci aveva presentato sulla lavagna la scheda elettorale col "sì" e tutti i bambini dovevano ricopiarla. Io invece, memore degli insegnamenti familiari, ho scritto "no". La maestra si è precipitata a distruggere le "prove" perché non venisse compromessa la mia famiglia. Nei paesi, infatti, fortunatamente, c'era una specie di catena di Sant'Antonio di solidarietà che attutiva il peggio.

Mio padre era insegnante di disegno, artista appassionato. Spesso egli usciva a dipingere en plein air e io non di rado lo accompagnavo, fin da piccolo. Mi sentivo esaltato, ma anche un po' sopraffatto dalla sua bravura (e forse ciò mi ha allontanato dal dipingere). Ma questa continua sollecitazione mi aveva creato un senso di omogeneità tra arte e natura. La natura era bella, mio padre la ritraeva, la riportava in casa; era tutto un pullulare di fattori di alta suggestione. Ricordo soprattutto colori, suoni, i paesaggi che lui dipingeva, le musichette comuni che risuonavano per le strade del paese e nella mia casa, grazie a improvvisati cantori e a vetusti grammofoni. Ricordo tutti gli elementi che mi affascinavano, che mi davano il senso di un possibile "modo di essere" più alto, migliore. E fin da bambino avvertivo quel canto interno della lingua che è la poesia, attraverso filastrocche e passi di poesiole (lette in casa o alle elementari) che scintillavano e tintinnavano nella loro essenza fonico-ritmica.

Posso dire che c'è in me un fondo idillico, ma che talune circostanze hanno attivato anche un fondo drammatico che mi ha portato, lungo il tempo, verso tensioni violentissime nel campo espressivo e quindi alla necessità della sperimentazione, di cercare nuove vie, diversi equilibri (o squilibri formali...). 
Del resto ho sofferto lutti familiari pesantissimi, da cui è derivato il senso di provvisorietà spaventosa che ha accompagnato la mia infanzia. Quando avevo circa sette anni è morta una mia sorellina che non ne aveva nemmeno sei, e la sua gemella morì anch'essa, sempre per malattie che oggi si sarebbero curate con poche iniezioni. La mortalità infantile era allora altissima. Durante l'ottobre a casa mia c'era un gran rigiro di angioletti di latta montati su croci ad asta e questi angioletti portavano il nome, il cognome e la data di nascita e morte dei bambini. Andavano a finire nell'angolo di cimitero ad essi riservato e le famiglie facevano dipingere o rinfrescare per i giorni della Commemorazione questi angioletti. Mio padre aveva un sovrappiù di lavoro di questo tipo, da cui ricavava poche lire, un po' di patate, frutta o fagioli. C'erano dunque nella mia infanzia degli elementi strani, inquietanti, come appunto quel lato del cimitero con le croci di latta che stridevano al vento e sembravano fatte apposta per la retorica della morte e dell'innocenza. Insomma non ho mai vissuto la natura come avrei voluto, con totale partecipazione, perché c'erano delle spine irritative sia all'interno della mia storia privata sia nell' ambito generale.

\section{Qual è il suo rapporto con il dialetto?}

Il mio rapporto con il dialetto è stato inconsapevole: era per me un dato naturale, appreso così, parlando in famiglia, nel gruppo, parlando come si respira. Solo più tardi ho preso consapevolezza della lingua, e poi delle lingue, aiutato da un certo plurilinguismo prodotto dai molti immigranti che ritornavano stagionalmente, e dalla presenza "mitica" del latino della chiesa. Di fatto, le persone più colte e scolarizzate parlavano anche l'italiano nelle situazioni formali e lo usavano scrivendo. Come lingua internazionale si aggiungeva poi il francese: si restava entro una fraternità neolatina.

\section{Ł̀ un abitudinario? Quando scrive meglio, al mattino o di sera?}

Io faccio ora la vita di un pensionato. Quando ho lasciato la scuola, dopo 35 anni d'insegnamento, avevo intenzione di spostarmi a Milano, dove ho un bugigattolo occupato ora dai figli. Ho cominciato invece ad essere tormentato da acciacchi abbastanza pesanti: disturbi psicosomatici, dolori persistenti e quindi l'accentuarsi di balordi elementi fobici che hanno ridotto il mio circuito di movimento. Tutto ciò si attenua solo se riesco a imboccare i vecchi filoni della mia preistoria affettiva che va verso il mito "ambientale", nonostante tutte le devastazioni che lungo i decenni, e specie qui nel Veneto, l'hanno sopraffatto. Devo faticare per ritrovare 
qui la mia geografia originale: tutto è cambiato, e anche questa è una causa di grande disagio. Ora non mi sento più dentro un paesetto ben articolato in mezzo ad altri paesetti, ma dentro uno sfilacciamento urbano e non urbano nello stesso tempo. Dovrei adattarmi all'idea che il Veneto è già una megalopoli inconsapevole di esserlo e che io ci vivo. Invece conservo ancora un'idea infantile degli spazi e non la rifiuto perché, infine, imboccare una stradina mai imboccata prima in tanti anni o trovare una valletta nuova è sempre una scoperta.

Tornando alla mia giornata, siccome il mio sonno è incerto, trascorro il mattino cercando di liberarmi delle nebbie dei sonniferi, con piccoli lavori poco impegnativi. Ma ci sono continuamente occasioni d'incontro: sia con vecchi amici, sia con circoli locali. E ci sono anche dei giovani che vengono ad aiutarmi a riordinare un po' le mie scartoffie.

Ho poi sempre un enorme arretrato di progetti non attuati da riprendere. Però il momento poetico viene, come si sa, quando viene, posso scrivere in qualsiasi momento, specie di notte. A volte scrivo magari cinquanta frammenti tutti di seguito. Ma mi piace anche molto indulgere ai diari-brogliacci dove annoto le mie fantasticherie di tipo vecchio e di tipo nuovo. Poi magari li elimino in buona parte. Essi servono soprattutto a farmi capire quanto sono scombinato, ma qualche volta funzionano da vecchi muriccioli su cui ha attecchito un seme. Ho una specie di attività da flâneur. Ma ritengo che per la poesia occorra lasciarsi trasportare come sugheri dalle acque. Per trovarsi magari nel pantano...

Lei, nella sua opera, cerca un linguaggio per questo tempo, la poesia possibile all'uomo di oggi, così turbato, inquieto, senza risposte, lacerato dalla guerra, dallo sfruttamento indiscriminato dell'ambiente, dal consumismo. Il suo sperimentalismo, le sue ricerche linguistico-strutturali non sono, pertanto, fini a se stesse, nel senso di un neovanguardismo gratuito e vuoto, ma sono un percorso essenziale, intimo e traumatico. Vuole descrivere un po' questo percorso?

L'idea dello sperimentalismo l'ho sempre implicitamente accettata perché non ho mai creduto a una poesia "immobile", pur avendo sempre davanti modelli classici, irrinunciabile luce ed enigma. Il mio era un andare avanti con molta incertezza, al contrario dei movimenti avanguardistici che avanzano un po' a carro armato, con forte apparato teorico. Io credevo alle amicizie, alle sintonie parziali, non ai gruppi. Il gruppo rappresentava per me la gestione di qualcosa di extraletterario, mentre io pensavo che ognuno dovesse seguire la sua strada e poi confrontarsi con gli altri. Già in anni lontani avevo elaborato l'idea del "convenzionismo", 
per cui ogni modello di scrittura o prospezione era da accettare soltanto come una "morale provvisoria", e per qualche aspetto falsa. In ciò si rispecchiava il diluvio di falsità che sentivo intorno a me, anche come fatto politico e sociale. L'incombere del cosiddetto "equilibrio del terrore" mi sembra trattenere l'intera realtà in un ossimoro paralizzante. E uno sgomentante disagio si sta protraendo nell'oggi, diverso, ma forse in peggio, da quella situazione: e basti pensare agli orrori dell'ex Jugoslavia. Per questo non può apparire che convenzionale, già nata falsa, qualunque innovazione.

D'altra parte l'accumulo esponenziale d'imprevisti di ogni genere e la trasformazione disordinata della realtà nei recenti decenni provocavano il senso di un amorfismo, di una derealizzazione sempre più soffocante. Nello sperimentalismo si rispecchiava tutto questo, e insieme si sviluppava un tentativo sempre frustrato di superarlo. Anche se appariva, talvolta, il lampo di un'innovazione positiva.

Comunque, la meditazione sul linguaggio e sulle sue impotenze (varie come le sue potenze), l'avvertire il linguaggio come qualcosa da mettere perpetuamente in questione, come uno strumento che dovesse mutare in continuazione per offrire qualche chance operativa - e nella realtà e nel lavoro poetico - hanno avuto per me una continua, intensa stimolazione, anche in situazioni limite.

Fin dalla primissima giovinezza mi ero soffermato su temi psicoanalitici, antropologici, linguistici come la convenzionalità e lo slittamento dei significati in rapporto ai segni, l'irreperibilità - quando si parla - di un "vero" e preciso referente esterno. Certe esperienze in vivo mi moltiplicavano all'infinito le durezze di quei temi. Da una parte esisteva il linguaggio immediato ed eterno della "natura" che non afferma eppure non nega: il suo avvolgimento mi si presentava come più dolce che tenebroso. Invece quando entrano in scena esseri umani, il linguaggio è sempre sul punto di non funzionare. Forse di là viene anche la mia primitiva tendenza a tener sotto mano modelli d'espressione "sicuri". E per queste vie andavano le mie meditazioni sul petèl, sul linguaggio infantile, risalenti agli anni quaranta, a quando non conoscevo affatto Jakobson. Mi sorprendevo a riprendere - anche ironicamente - il gusto del bamboleggiare con linguaggi pseudo-infantili, con dialetti inventati o effettivamente usati, magari col gatto di casa. E, appunto, non perdevo di vista la tradizione, che, in un certo senso, poteva sovrapporsi senza difficoltà alla natura perché ormai era tale da apparire emanazione di una sapienza della natura stessa... 


\section{Secondo Lei, quali sono i rapporti fra storia, storiografia e poesia?}

Continuo a pensare che non esista storiografia più precisa della poesia, se essa viene correttamente interpretata, perché nel suo corpo "ectoplastico" si infiltrano tutte le più acuminate realtà della storia e in esso si esprimono, anche (soprattutto) negli aspetti formali.

Noi sappiamo di più, forse, della realtà del mondo antico attraverso un frammento di vissuto "salvato" dalla poesia che attraverso la ricerca archeologica.

L'ultimo gradino dell'essenza, l'ultimo distillato del vivere si accompagna con questo andirivieni della poesia e corrisponde ad esso. Resta tuttavia la compresenza, per me, di un mito, quello di una certa poësis perennis, cioè immune dal tempus edax, che si sgranocchia via tutto. Del resto, si sa che anche le lingue muoiono, e con esse la verità più intima dei messaggi che esse hanno portato.

\section{Che cosa pensa del momento difficile che viviamo, momento d'imbarbarimento quasi} generalizzato? La poesia può fare qualcosa, può darci speranza?

Oggi non si sa bene più cosa sia l'uomo. Fino a non molto tempo fa si credeva di saperne molto, sull'essenza dell'umano, su ciò che fosse la vera humanitas, e su quel frutto dell'evoluzione, tutto sommato positivo, che l'uomo appariva. Durante il secondo dopoguerra, tuttavia, e soprattutto negli anni del riarmo atomico, che dava una soffocante carica di nonsenso generale al "dato umano", si è molto diffusa una specie di sfiducia globale dell'uomo verso se stesso. Si tentava di cancellarla con l'attivismo di uno sviluppo economico e di un presunto progresso politico, che poi si è ridotto a poca cosa, anche se non si può negare che vi sia stato qualche filo lucente di positività. A distanza di molto tempo, ormai, dai primi anni del dopoguerra il bilancio si rivela deludente, perché l'uomo sembra vivere soltanto di residui d'ideologie, marcate da forti interrogativi, anche se non annullate del tutto (come molti pensano) e minate da una violenta "carica" nichilista. L'uomo sembra assumere in proprio il sentimento dell'entropia in atto. Cosa può la poesia in un simile quadro, pur nascendo dai sottofondi più celati della vita? Forse potrebbe dire qualcosa di valido per l'umanità, ma non esiste il parametro per definire questo "qualcosa". E sapere almeno qualcosa sul proprio senso (destino?) è necessario. L'uomo sta ribollendo nel proprio enigma, e la poesia non può dare che dei lampi di "consolazione", nei quali appare ancora il miraggio dell'autofondazione e dell'autogiustificazione dell'essere. In essa c'è dunque un qualche valore, almeno provvisorio. Ma il quadro che abbiamo di fronte è quello di una catastrofe "ecologica” della mente (ricorderei qui Bateson): l'urgenza di una visione 
ecologica del mondo si è resa necessaria nel momento in cui si è concretizzata la minaccia apportata all'ecosistema in generale da quel piccolo, terribile sistema che diciamo uomo.

La poesia continua (per ora!) a dare il suo bip-bip che poco presume ma si sente non tacitabile. E chissà che non vi sia chi lo coglie. Bisognerebbe poi ricordare (insieme con i Surrealisti) che la poesia dovrebbe essere fatta da tutti, non da uno. 